\title{
口m
}

BioMedical Engineering OnLine



The effect of baseline pressure errors on an intracranial pressure-derived index: results of a prospective observational study

Eide et al. 


\section{The effect of baseline pressure errors on an intracranial pressure-derived index: results of a prospective observational study}

Per Kristian Eide ${ }^{1,2^{*}}$, Angelika Sorteberg ${ }^{1}$, Torstein R Meling ${ }^{1}$ and Wilhelm Sorteberg ${ }^{1}$

\section{* Correspondence:} p.k.eide@medisin.uio.no

${ }^{1}$ Department of Neurosurgery, Oslo University Hospital, Rikshospitalet,

Oslo, Norway

${ }^{2}$ Faculty of Medicine, University of

Oslo, Oslo, Norway

\begin{abstract}
Background: In order to characterize the intracranial pressure-volume reserve capacity, the correlation coefficient (R) between the ICP wave amplitude (A) and the mean ICP level (P), the RAP index, has been used to improve the diagnostic value of ICP monitoring. Baseline pressure errors (BPEs), caused by spontaneous shifts or drifts in baseline pressure, cause erroneous readings of mean ICP. Consequently, BPEs could also affect ICP indices such as the RAP where in the mean ICP is incorporated.
\end{abstract}

Methods: A prospective, observational study was carried out on patients with aneurysmal subarachnoid hemorrhage (aSAH) undergoing ICP monitoring as part of their surveillance. Via the same burr hole in the scull, two separate ICP sensors were placed close to each other. For each consecutive 6-sec time window, the dynamic mean ICP wave amplitude (MWA; measure of the amplitude of the single pressure waves) and the static mean ICP, were computed. The RAP index was computed as the Pearson correlation coefficient between the MWA and the mean ICP for 406 -sec time windows, i.e. every subsequent 4-min period (method 1). We compared this approach with a method of calculating RAP using a 4-min moving window updated every 6 seconds (method 2).

Results: The study included 16 aSAH patients. We compared 43,653 4-min RAP observations of signals 1 and 2 (method 1), and 1,727,000 6-sec RAP observations (method 2). The two methods of calculating RAP produced similar results. Differences in RAP $\geq 0.4$ in at least $7 \%$ of observations were seen in 5/16 (31\%) patients. Moreover, the combination of a RAP of $\geq 0.6$ in one signal and $<0.6$ in the other was seen in $\geq 13 \%$ of RAP-observations in 4/16 (25\%) patients, and in $\geq 8 \%$ in another $4 / 16$ (25\%) patients. The frequency of differences in RAP $>0.2$ was significantly associated with the frequency of BPEs $(5 \mathrm{mmHg} \leq \mathrm{BPE}<10 \mathrm{mmHg})$.

Conclusions: Simultaneous monitoring from two separate, close-by ICP sensors reveals significant differences in RAP that correspond to the occurrence of BPEs. As differences in RAP are of magnitudes that may alter patient management, we do not advocate the use of RAP in the management of neurosurgical patients. 


\section{Background}

Monitoring of intracranial pressure (ICP) is crucial in the intensive care management of neurosurgical patients [1-4]. The common management goal is to maintain the static pressure parameter mean ICP $<20-25 \mathrm{mmHg}$ [3]. When the cardiac-induced ICP waves are monitored as well, the goal is to keep the dynamic pressure parameter mean ICP wave amplitude (MWA) $<5 \mathrm{mmHg}$ [1]. In an attempt to better characterize the intracranial pressure-volume reserve capacity, some authors have also calculated an index measuring the correlation coefficient $(\mathrm{R})$ between the ICP wave amplitude (A) and the mean ICP level (P), denoted RAP $[5,6]$. Repetitive computation of this RAP index has been used in the surveillance of patients with traumatic brain injury (TBI), cerebral bleeds, and hydrocephalus [6-9]. Using the RAP index, which ranges from -1 to +1 , the upper normal threshold is +0.6 [6,8-12]. A RAP approaching +1 has hence been considered as indicative of reduced compensatory reserve capacity, or impaired intracranial compliance.

The mean ICP is always determined against a baseline pressure (i.e. zero or reference pressure). A major weakness of this is that the mean ICP value that is displayed to the physician or nurse becomes erroneous if this baseline pressure is spontaneously altered during ongoing monitoring [13]. Such baseline pressure errors (BPEs) occur during clinical ICP monitoring, and has been seen in a variety of ICP sensor types and locations from where the ICP is recorded [13,14]. In contrast, the dynamic ICP parameters, such as the MWA remain unaffected by BPEs as they express just changes of ICP within each heartbeat.

In a recent prospective, observational study on 16 patients with aneurysmal subarachnoid haemorrhage (SAH), we reported a high frequency and severity of BPEs [15]. Being recognized as differences in mean ICP in combination with close to identical MWA, BPEs affect the RAP index, causing the RAP to differ widely when determined from two separate ICP sensors in the same patient [16]. This is most evident when monitoring ICP from two different types of ICP sensors [16]. The present study was undertaken to explore the frequency and severity of differences in RAP when using two ICP sensors of the same type. To this end, we reanalysed the continuous ICP recordings of 16 aSAH patients enrolled in a prospective, observational study in which we recorded the ICP from two separate ICP sensors placed in the brain tissue via the same burr hole in the scull [15].

\section{Methods}

\section{Patients}

Aneurysmal SAH patients who need continuous ICP monitoring as part of their intensive care management were enrolled in the study. Their intensive care management was not influenced by their participation.

The study was approved by The Regional Ethics Committee, REK South-East (2010/ 1328B) and Oslo University Hospital (2010/16315), Oslo, Norway. Inclusion was by oral and written informed consent, either by the patient herself/himself or by the closest family member.

\section{Study design}

A prospective, observational study design was used to determine the frequency and severity of BPEs, and how BPEs affect scores of ICP and ICP indices. These data were 
re-analyzed in order to investigate the frequency and severity of differences in RAP. Moreover, we compared two different methods of calculating the RAP.

\section{Monitoring and analysis of ICP}

Two Raumedic NeuroVent P (Raumedic AG, Münchberg, GE) ICP sensors were placed in the brain parenchyma via the same burr hole, either during aneurysm surgery, or during placement of an external ventricular drain (EVD). The location of the ICP sensors was verified postoperatively by cerebral computer tomography $(\mathrm{CT})$ scanning.

Both sensors were connected to the MPR-1 monitor (Raumedic AG, Münchberg, GE), which in turn was connected to a laptop computer running Sensometrics Software (dPCom AS, Oslo, Norway). The digital sampling rate of pressure signals from the two sensors (Sensors 1 and 2) was $100 \mathrm{~Hz}$. The raw data files were stored on the computer. Recordings from the two sensors continued throughout the time period the patient was in need of ICP surveillance.

As previously described, the ICP signals were analyzed according to the methodology implemented in Sensometrics Software (Figure 1) [17]. An automatic method identifies the heartbeat-induced single ICP waves, differentiating them from the pressure waves of other origins (noise or various artifacts). Each heartbeat-induced single ICP wave is characterized by the following wave parameters: the amplitude $(\mathrm{dP})$, rise time $(\mathrm{dT})$, and the rise-time coefficient ( $\mathrm{dP} / \mathrm{dT}$ ) (Figure 1c). Only 6-sec time windows containing a minimum of four cardiac-beat-induced waves were included for further analysis. The MWA is computed in consecutive 6-sec time window (Figure 1c). During the same 6-sec time windows, the mean ICP is determined as the sum of sample values divided by the number of samples.

\section{Calculation of RAP}

The software incorporates an automatic procedure for determining the correlation coefficient (R) between the ICP wave amplitude (A) and the ICP level (P), the RAP, during consecutive 4-min time periods. The RAP-index is the Pearson correlation coefficient between the MWA and the mean ICP during 40 6-sec time window periods. Computation of RAP has previously been described by others [6,12]. Since we compared the RAP of two simultaneous ICP signals, the RAP of Sensors 1 and 2 were derived from simultaneous 6 -sec time windows (Figures $1 \mathrm{a}-\mathrm{b}$ ).

The Pearson correlation coefficient is a measure of the strength of a relationship between two variables, ranging from -1 to +1 . When one variable changes in the opposite direction of the other, the correlation coefficient becomes negative, whereas the correlation coefficient becomes positive when both variables change in the same direction (Figures 1d-f). The closer the correlation coefficient is to + or -1 , the stronger is the relationship between the two variables. The assumptions for using the Pearson correlation coefficient during 4-min periods as performed in this study were fulfilled: Both the mean ICP and the MWA are continuous and independent observations that follow a normal distribution. Moreover, for intervals of 4-minute duration, the correlation coefficient between these two observations reflects a linear relationship. 
(a)

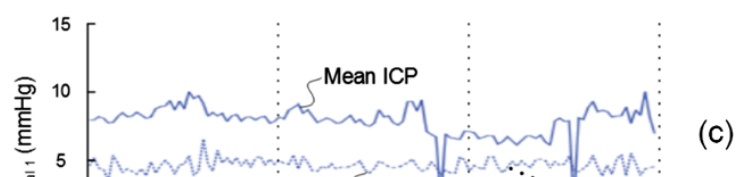

(d)

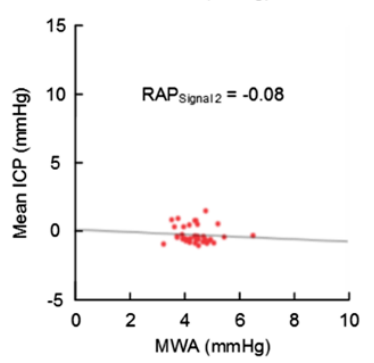

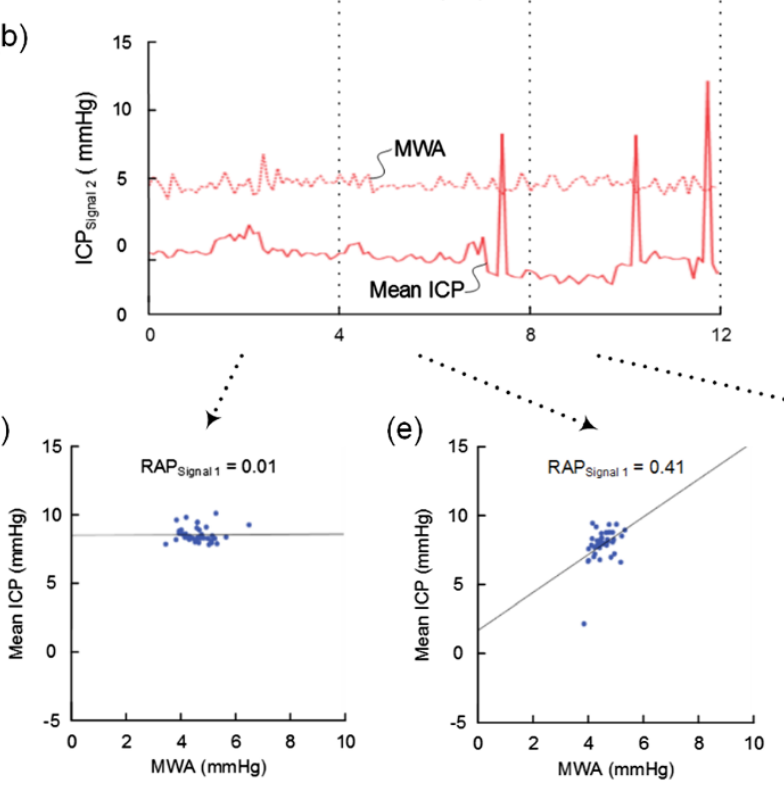

(c)

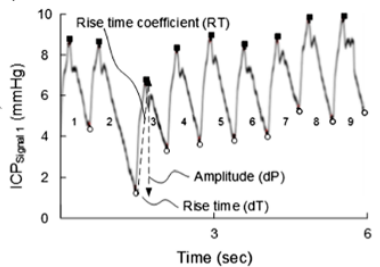

(b)


Figure 1 Illustration of the method of determining the RAP [correlation coefficient (R) between the intracranial pressure (ICP) wave amplitude (A) and the mean ICP level (P)] in patient 2. Following automatic identification of the cardiac induced single intracranial pressure (ICP) waves, the mean ICP and mean wave amplitude (MWA) are determined for every consecutive 6-sec time window. Trend plots of mean ICP and MWA determined during the same 6-sec time windows are shown for Signals 1 (a) and 2 (b) over subsequent 12 min periods (representing three 4-min periods, and 120 6-sec time windows). For Signal 1 (a) the average ( \pm standard deviation) of mean ICP was $7.9 \pm 1.2 \mathrm{mmHg}$ and of MWA $4.6 \pm 0.5 \mathrm{mmHg}$; while for Signal 2 (b) mean ICP $-0.9 \pm 1.9 \mathrm{mmHg}$ and MWA $4.4+0.5 \mathrm{mmHg}$ (mean difference of ICP $-8.8 \pm 2.2 \mathrm{mmHg}$; mean difference of MWA $-0.2 \pm 0.2 \mathrm{mmHg}$ ). In (c) one single 6 -sec time window is shown, demonstrating the individual single ICP waves, each wave being characterized by the amplitude (dP), rise time (dT), and rise time coefficient (RT) (indicated for single wave 3). RAP is determined as the Pearson correlation coefficient between MWA and the mean ICP during subsequent 4 min periods (representing 40 6-sec time windows). In the patients included in this study, we compared the RAP values during identical 4-min periods for Signals 1 and 2 (referred to as method 1). This was performed for every consecutive 4-min period. For the three consecutive 4-min periods shown in (a) and (b), the corresponding scatter plots and RAP-values are presented in (d), (e), and (f), respectively. The differences in RAP were associated with marked differences in mean ICP between Signals 1 and 2 whereas the MWA was close to identical. 
We further compared two different methods of calculating the RAP:

(i) Method 1. According to method 1, a new RAP value was calculated every 4 min period. Hence, for every consecutive 4-min period the software determined the Pearson correlation coefficient (RAP) values of the two ICP signals (Figures 1c-d). The RAP scores could then be trended as shown in Figure 2.

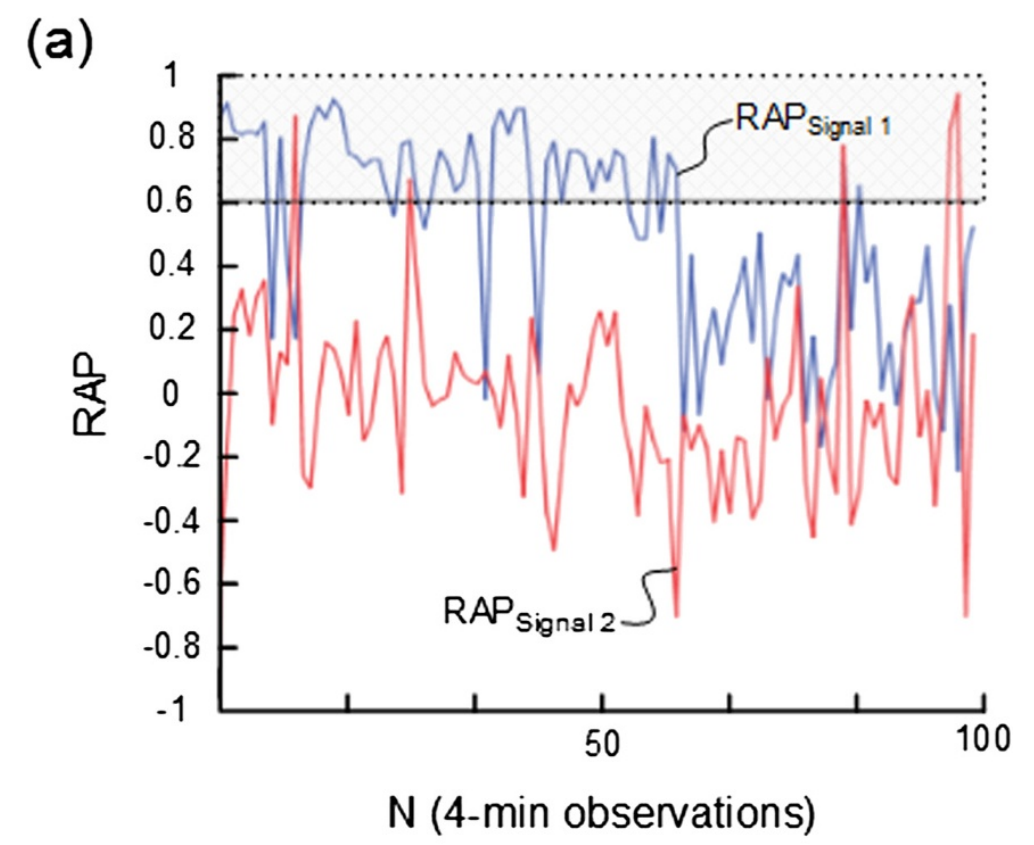

(b)

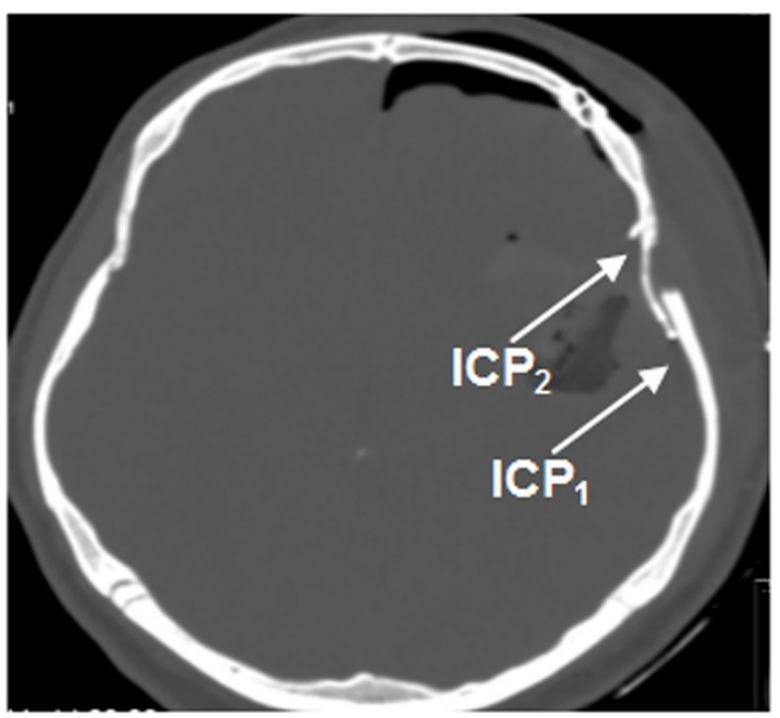

Figure 2 Trend plots of RAP [correlation coefficient (R) between the intracranial pressure (ICP) wave amplitude (A) and the mean ICP level (P)] of Signals 1 and 2 in patient 2. For patient 2, the trend plots of (a) RAP determined during 100 consecutive 4-min periods for signals 1 (blue line) and 2 (red line) show marked differences (average of RAP signal $_{1}$ 0.50; average of RAP Signal $2_{2}-0.04$ ). The horizontal lines at RAP 0.6 illustrate a commonly used upper normal threshold for RAP. The intracranial locations of the ICP sensors 1 and 2 are illustrated in (b). 
(ii) Method 2. This method incorporates a moving time window. A new RAP value is updated every 6 second. Consequently, every 6 second, the 'oldest' averaged value drops out and the 'newest' drops in. This approach was previously described by others [18], except that in this latter study the authors used 5 min intervals and 10 seconds windows, as compared to 4 min intervals and 6 seconds windows in the present paper.

\section{Determination of baseline pressure errors (BPEs)}

BPEs were defined as differences in mean ICP in combination with close to identical MWAs (difference between sensors $<0.5 \mathrm{mmHg}$ ), as recently described in this journal [15]. In this study, we determined the percentage of recording time with BPEs of given magnitudes $(5 \mathrm{mmHg} \leq \mathrm{BPE}<10 \mathrm{mmHg}$ ) for every patient.

\section{Results}

Patients

The study enrolled a total of 16 aSAH patients, 8 females and 8 males, with median age 58 (ranges 39 - 74) years (Table 1, left). The distances between the two ICP sensors are presented in Table 1, right and were: axially median 11 (ranges 2-38) mm, coronally median 8 (ranges 3-20) $\mathrm{mm}$ and sagitally median 9 (ranges 3-26) $\mathrm{mm}$. No adverse effects of the ICP monitoring were observed.

\section{Method 1: comparison of 4-min RAP-values between signals 1 and 2}

Table 2 left shows the number of 4-min RAP observations obtained in the 16 patients. For all patients combined, 43,653 4-min RAP-values were available for analysis; median number for the patients was 2,472 (ranges 1,090 - 5,522).

Table 1 Demographic data of the 16 patients included in the study, and distance between Sensors 1 and 2

\begin{tabular}{|c|c|c|c|c|c|}
\hline \multirow[t]{2}{*}{ Pat } & \multirow[t]{2}{*}{ Age } & \multirow[t]{2}{*}{ Gender } & \multicolumn{3}{|c|}{ Distance between ICP Sensors 1 and $2(\mathrm{~mm})$} \\
\hline & & & Axial & Coronal & Sagittal \\
\hline 1 & 52 & $M$ & 13 & 9 & 7 \\
\hline 2 & 57 & M & 30 & 17 & 22 \\
\hline 3 & 64 & M & 6 & 17 & 11 \\
\hline 4 & 59 & $\mathrm{~F}$ & 3 & 5 & 11 \\
\hline 5 & 58 & $\mathrm{~F}$ & 38 & 13 & 25 \\
\hline 6 & 39 & $\mathrm{~F}$ & 13 & 11 & 7 \\
\hline 7 & 58 & $\mathrm{~F}$ & 11 & 6 & 18 \\
\hline 8 & 66 & $M$ & 4 & 5 & 5 \\
\hline 9 & 44 & $M$ & 3 & 3 & 3 \\
\hline 10 & 70 & $M$ & 7 & 4 & 5 \\
\hline 11 & 49 & $M$ & 11 & 5 & 6 \\
\hline 12 & 52 & $\mathrm{~F}$ & 21 & 20 & 26 \\
\hline 13 & 63 & $\mathrm{~F}$ & 2 & 4 & 4 \\
\hline 14 & 74 & $\mathrm{~F}$ & 13 & 11 & 11 \\
\hline 15 & 56 & $F$ & 3 & 3 & 9 \\
\hline 16 & 55 & $M$ & 19 & 19 & 8 \\
\hline Median (Ranges) & $58(39-74)$ & $8 F-8 M$ & $11(2-38)$ & $8(3-20)$ & $9(3-26)$ \\
\hline
\end{tabular}


Table 2 Comparison of 4-min RAP-values between Signals 1 and 2

\begin{tabular}{|c|c|c|c|c|c|c|}
\hline \multirow[t]{2}{*}{ PatID } & \multirow[t]{2}{*}{ N (4-min RAP observations) } & \multicolumn{2}{|c|}{ RAP (average + std) } & \multicolumn{3}{|c|}{$\begin{array}{l}\text { Differences in RAP between } \\
\text { Signals } 1 \text { and } 2(\mathrm{~N}, \%)\end{array}$} \\
\hline & & Signal 1 & Signal 2 & 0.2 & 0.4 & 0.6 \\
\hline 1 & 1,850 & $0.23 \pm 0.43$ & $0.26 \pm 0.42$ & $483(26 \%)$ & $147(8 \%)$ & $51(3 \%)$ \\
\hline 2 & 1,090 & $0.15 \pm 0.36$ & $-0.01 \pm 0.36$ & $612(56 \%)$ & $338(31 \%)$ & $179(16 \%)$ \\
\hline 3 & 4,706 & $0.61 \pm 0.33$ & $0.62 \pm 0.32$ & $365(8 \%)$ & $72(2 \%)$ & $30(1 \%)$ \\
\hline 4 & 2,119 & $0.36 \pm 0.38$ & $0.36 \pm 0.38$ & $200(9 \%)$ & $29(1 \%)$ & 8 \\
\hline 5 & 1,651 & $0.60 \pm 0.35$ & $0.61 \pm 0.35$ & $53(3 \%)$ & $11(1 \%)$ & 3 \\
\hline 6 & 1,119 & $0.42 \pm 0.35$ & $0.43 \pm 0.35$ & $187(17 \%)$ & $54(5 \%)$ & $10(1 \%)$ \\
\hline 7 & 3,209 & $0.65 \pm 0.30$ & $0.68 \pm 0.28$ & $236(7 \%)$ & $83(3 \%)$ & $35(1 \%)$ \\
\hline 8 & 3,747 & $0.51 \pm 0.39$ & $0.35 \pm 0.41$ & $1,311(35 \%)$ & $806(22 \%)$ & $456(12 \%)$ \\
\hline 9 & 4,221 & $0.53 \pm 0.40$ & $0.61 \pm 0.34$ & $982(23 \%)$ & $506(12 \%)$ & $323(8 \%)$ \\
\hline 10 & 3,538 & $0.25 \pm 0.33$ & $0.26 \pm 0.33$ & $166(5 \%)$ & $20(1 \%)$ & 11 \\
\hline 11 & 1,494 & $0.62 \pm 0.32$ & $0.63 \pm 0.31$ & $54(4 \%)$ & $14(1 \%)$ & 4 \\
\hline 12 & 3,089 & $0.83 \pm 0.24$ & $0.83 \pm 0.25$ & $19(1 \%)$ & 3 & 2 \\
\hline 13 & 1,381 & $0.28 \pm 0.34$ & $0.32 \pm 0.35$ & $413(30 \%)$ & 113 (8\%) & $26(2 \%)$ \\
\hline 14 & 2,093 & $0.38 \pm 0.34$ & $0.37 \pm 0.35$ & $254(12 \%)$ & $72(3 \%)$ & $31(1 \%)$ \\
\hline 15 & 5,522 & $0.52 \pm 0.36$ & $0.50 \pm 0.37$ & 729 (13\%) & 142 (3\%) & $28(1 \%)$ \\
\hline 16 & 2,824 & $0.38 \pm 0.36$ & $0.39 \pm 0.36$ & 393 (14\%) & 73 (3\%) & $15(1 \%)$ \\
\hline
\end{tabular}

RAP: correlation coefficient (R) between the intracranial pressure (ICP) wave amplitude (A) and the mean ICP level (P).

Figure 1 illustrates how differences in RAP between Signals 1 and 2 were associated with marked differences in mean ICP whereas the MWAs remained close to identical during the recording.

Simultaneous 4-min RAP scores (determined according to method 1) from Signal 1 and Signal 2 are presented in Table 2. While Table 2, middle, lists the RAP scores (mean and \pm standard deviation) for each patient, Table 2, right presents differences in RAP between the two signals that were $\geq 0.2, \geq 0.4$, and $\geq 0.6$, respectively. Major differences $(\geq 0.4)$ in RAP were seen in $5(31 \%)$ of 16 patients, including patients $1,2,8,9$, and 13 (Table 2, right). The trend plots of RAP of the two signals are visualized for subjects 2, 8, and 9 in Figures 2a, 3a, and 4a, respectively. The locations of the ICP sensors for these three patients are shown on CT scans in Figures $2 \mathrm{~b}, 3 \mathrm{~b}$, and $4 \mathrm{~b}$, respectively.

Table 3 presents the portion of 4-min observations wherein RAP was $<0.6$ in both signals (left), $\geq 0.6$ in both signals (middle) and $<0.6$ in one signal and $\geq 0.6$ in the other (right). The combination of a RAP of $<0.6$ in one signal and $\geq 0.6$ in the other was seen in $\geq 14 \%$ of scores in $4 / 16(25 \%)$ patients (patients $2,8,9$ and 13 ), and in $\geq 9 \%$ of observations in another $4 / 16$ (25\%) patients) (patients $3,6,7$, and 15).

\section{Method 2: comparison of 6-sec RAP-values between signals 1 and 2}

The 6-sec RAP observations of the 16 patients are shown in Table 4. A total of 1,727,000 6-sec RAP values were analyzed; median number for the 16 patients was 97,922 (ranges 42,162 - 220,276). Table 4, right shows differences in RAP between the two signals that were $\geq 0.2, \geq 0.4$, and $\geq 0.6$, respectively. Major differences $(\geq 0.4)$ in RAP were seen in 5 of 16 patients (31\%), including patients $1,2,8,9$, and 13 (Table 4, right). 




(b)

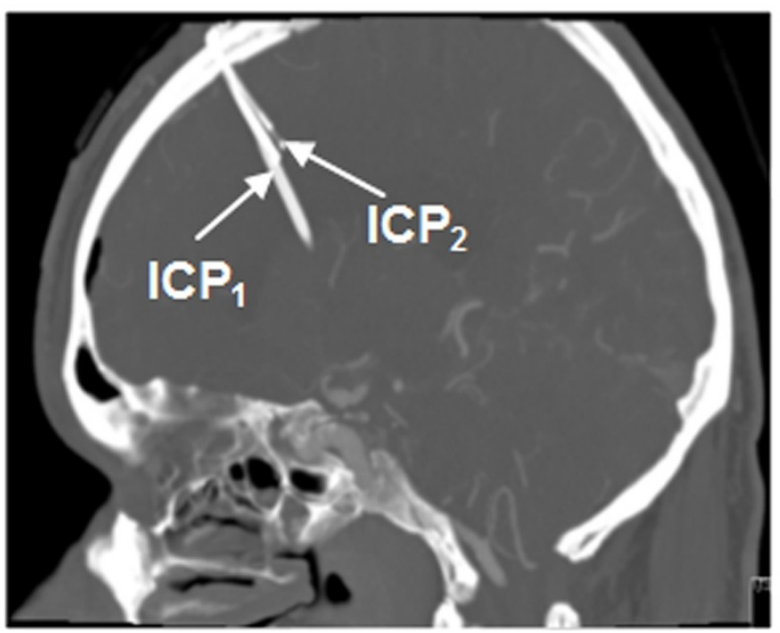

Figure 3 Trend plots of RAP [correlation coefficient (R) between the intracranial pressure (ICP) wave amplitude (A) and the mean ICP level (P)] of Signals 1 and $\mathbf{2}$ in patient $\mathbf{8}$. For patient 8 the trend plots of (a) RAP determined during 100 consecutive 4-min periods for signals 1 (blue line) and 2 (red line) show marked differences (average of RAP signal 1 0.64; average of RAP signal 2 0.16). The horizontal lines at RAP 0.6 illustrate a commonly used upper normal threshold for RAP. The intracranial locations of the ICP sensors 1 and 2 are shown in (b).

Figure 5 illustrates that computation of RAP according to methods 1 and 2 gave close to identical results, as illustrated by the percentage of differences in RAP between signals 1 and $2 \geq 0.4$.

Table 5 presents the portion of 6 -sec observations wherein RAP was $<0.6$ in both signals (left), $\geq 0.6$ in both signals (middle) and $<0.6$ in one signal and $\geq 0.6$ in the other (right). The combination of a RAP of $<0.6$ in one signal and $\geq 0.6$ in the other in $\geq 8 \%$ of 


\section{(a)}



(b)

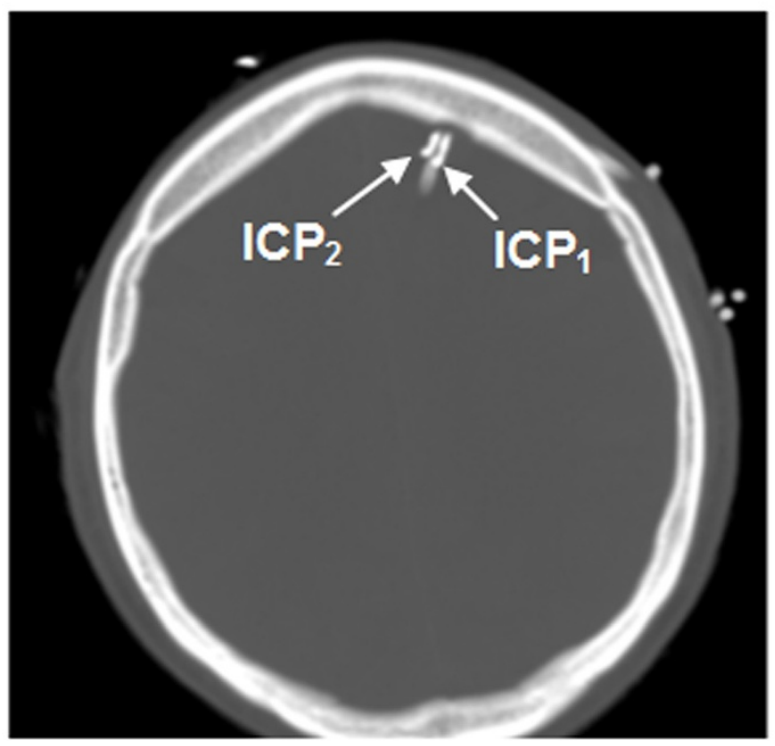

Figure 4 Trend plots of RAP [correlation coefficient (R) between the intracranial pressure (ICP) wave amplitude (A) and the mean ICP level (P)] of Signals 1 and 2 in patient $\mathbf{9}$. For patient 9 the trend plots of (a) RAP determined during 100 consecutive 4-min periods for signals 1 (blue line) and 2 (red line) show marked differences (average of RAP signal 10.17 ; average of RAP signal $2_{2.59}$ ). The horizontal lines at RAP 0.6 illustrate a commonly used upper normal threshold for RAP. The intracranial locations of the ICP sensors 1 and 2 are shown in (b).

observations was seen 8/16 (50\%) patients (patients 2, 3, 6-9, 13 and 15). Figure 6 further illustrates that the two different methods of calculating the RAP gave similar results regarding the percentage of observations where RAP was $<0.6$ in one signal and $\geq 0.6$ in the other. 
Table 3 Proportion of 4-min observations with RAP $<0.6$ in both signals (left), RAP $\geq 0.6$ in both signals (middle) and RAP $\geq 0.6$ in one signal while RAP $<0.6$ in another signal (right)

\begin{tabular}{|c|c|c|c|}
\hline PatID & $\begin{array}{l}\text { RAP }_{\text {SIGNAL } 1}<0.6 / \\
\text { RAP }_{\text {SIGNAL } 2}<0.6\end{array}$ & $\begin{array}{l}\operatorname{RAP}_{\text {SIGNAL } 1} \geq 0.6 / \\
\operatorname{RAP}_{\text {SIGNAL } 2} \geq 0.6\end{array}$ & $\begin{array}{c}\text { RAP }_{\text {SIGNAL } 1} \geq 0.6 / \text { RAP }_{\text {SIGNAL } 2}<0.6 \\
\text { or } \\
\text { RAP }_{\text {SIGNAL } 1_{1}<0.6 / \text { RAP }_{\text {SIGNAL } 2} \geq 0.6}\end{array}$ \\
\hline 1 & $1,360(73.5 \%)$ & $369(20 \%)$ & $121(6.5 \%)$ \\
\hline 2 & $910(83.5 \%)$ & $19(1.7 \%)$ & $161(14.8 \%)$ \\
\hline 3 & $1,521(32 \%)$ & 2,776 (59\%) & 409 (9\%) \\
\hline 4 & $1,371(64.7 \%)$ & $624(29.4 \%)$ & 124 (5.9\%) \\
\hline 5 & 547 (33.1\%) & $1,017(61.6 \%)$ & 87 (5.3\%) \\
\hline 6 & $652(58 \%)$ & $368(33 \%)$ & $99(9 \%)$ \\
\hline 7 & 779 (24\%) & 2,148 (67\%) & $282(9 \%)$ \\
\hline 8 & $45.6(45 \%)$ & 1,231 (32.9\%) & 809 (21.6\%) \\
\hline 9 & 1,498 (35.5\%) & $2,051(48.6 \%)$ & 672 (15.9\%) \\
\hline 10 & $2,800(79.1 \%)$ & 598 (16.9\%) & $140(4 \%)$ \\
\hline 11 & 521 (34.9\%) & 891 (59.6\%) & $82(5.5 \%)$ \\
\hline 12 & $323(10.5 \%)$ & 2,719 (88.0\%) & 47 (1.5\%) \\
\hline 13 & 974 (70\%) & $217(16 \%)$ & 190 (14\%) \\
\hline 14 & $1,349(64.5 \%)$ & 585 (28\%) & 159 (7.6\%) \\
\hline 15 & 2,619 (47\%) & 2,425 (44\%) & 478 (9\%) \\
\hline 16 & $1,798(63.7 \%)$ & 830 (29.4\%) & $196(6.9 \%)$ \\
\hline
\end{tabular}

RAP: correlation coefficient (R) between the intracranial pressure (ICP) wave amplitude (A) and the mean ICP level (P).

BPEs of various magnitudes versus difference in RAP

Figure 7 presents the correlation between percentages of BPEs of given magnitude ( $5 \mathrm{mmHg} \leq \mathrm{BPE}<10 \mathrm{mmHg}$ ) and the percentage of 4-min periods with RAP difference being $\geq 0.2$ (Figure 7a), $\geq 0.4$ (Figure $7 b$ ), and $\geq 0.6$ (Figure 7c). The correlation plots demonstrate that in patient recordings with a high percentage of BPEs, there is also a high percentage of marked differences in RAP, i.e. the occurrences of differences in RAP are associated with the occurrences of BPEs.

\section{Discussion}

The main observation of this study was a marked difference in RAP indices [moving correlation coefficient (R) between the ICP wave amplitude (A) and the ICP (P)] obtained from simultaneous registrations from two separate, close-by ICP sensors of the same type. The discrepancy in RAP between signals 1 and 2 was independent of the use of a moving window with frequent updates. The occurrences of differences in RAP were associated with occurrences of baseline pressure errors (BPEs). While the number of patients was rather small $(\mathrm{n}=16)$, the number of RAP observations was high (total 43,653 4-min RAP observations and 1,727,000 6-sec RAP observations), with a median number of RAP-observations for each patient (median 2,472).

\section{Clinical use of ICP-derived indices}

ICP derived indices were introduced in the early 1990's to enhance the diagnostic information of ICP monitoring [6,12]. In particular, the RAP index has been used as a possible indicator of the intracranial pressure volume compensatory reserve capacity 
Table 4 Differences in 6-sec RAP-values between Signals 1 and 2

\begin{tabular}{|c|c|c|c|c|}
\hline \multirow[t]{2}{*}{ Pat ID } & \multirow[t]{2}{*}{ N (6-sec RAP observations) } & \multicolumn{3}{|c|}{ Differences in RAP between Signals 1 and $2(N, \%)$} \\
\hline & & 0.2 & 0.4 & 0.6 \\
\hline 1 & 73,005 & $18,935(26 \%)$ & $5,907(8 \%)$ & $1,981(3 \%)$ \\
\hline 2 & 42,162 & $24,602(58 \%)$ & $13,710(33 \%)$ & $7,173(17 \%)$ \\
\hline 3 & 206,708 & $17,604(9 \%)$ & $4,773(2 \%)$ & $1,793(1 \%)$ \\
\hline 4 & 78,874 & $6,387(8 \%)$ & $938(1 \%)$ & 255 \\
\hline 5 & 43,936 & $1,162(3 \%)$ & $250(1 \%)$ & 81 \\
\hline 6 & 43,888 & $8,145(19 \%)$ & $2,288(5 \%)$ & $727(2 \%)$ \\
\hline 7 & 126,768 & $8,545(7 \%)$ & $2,939(2 \%)$ & $1,205(1 \%)$ \\
\hline 8 & 149,625 & $52,031(35 \%)$ & $31,938(21 \%)$ & $17,830(12 \%)$ \\
\hline 9 & 167,244 & $37,871(23 \%)$ & $19,370(12 \%)$ & 11,763 (7\%) \\
\hline 10 & 141,358 & $6,485(5 \%)$ & $1,015(1 \%)$ & 345 \\
\hline 11 & 59,520 & 2,205 (4\%) & $527(1 \%)$ & 161 \\
\hline 12 & 123,382 & $742(1 \%)$ & 127 & 57 \\
\hline 13 & 54,411 & $15,663(29 \%)$ & $4,065(7 \%)$ & $1,069(2 \%)$ \\
\hline 14 & 83,498 & 9,689 (12\%) & $2,793(3 \%)$ & $1,142(1 \%)$ \\
\hline 15 & 220,276 & $31,022(14 \%)$ & $7,126(3 \%)$ & $1,677(1 \%)$ \\
\hline 16 & 112,345 & 14,299 (13\%) & $2,657(2 \%)$ & $601(1 \%)$ \\
\hline
\end{tabular}

$[5,7,10,12]$. Several authors have found the RAP to be of value in the surveillance of patients with TBI $[6,9,10,12,19]$ and in the diagnostic assessment of hydrocephalus $[7,8,11]$; then proposing an upper normal threshold value of about $+0.6[6,8-11]$. However, the clinical usefulness of this index remains to be determined [20-22].

In contrast, as compared to RAP scores derived from amplitudes computed using the frequency domain method [6,12], we presently used an amplitude computed from the time domain method (the MWA) [17]. The frequency- and time-domain methods for calculating single pressure wave amplitudes are not equivalent [23]. The time domain method has the advantage of correctly identifying the heartbeat-induced pressure waves versus artifact waves. Using the frequency domain method, on the other hand, the

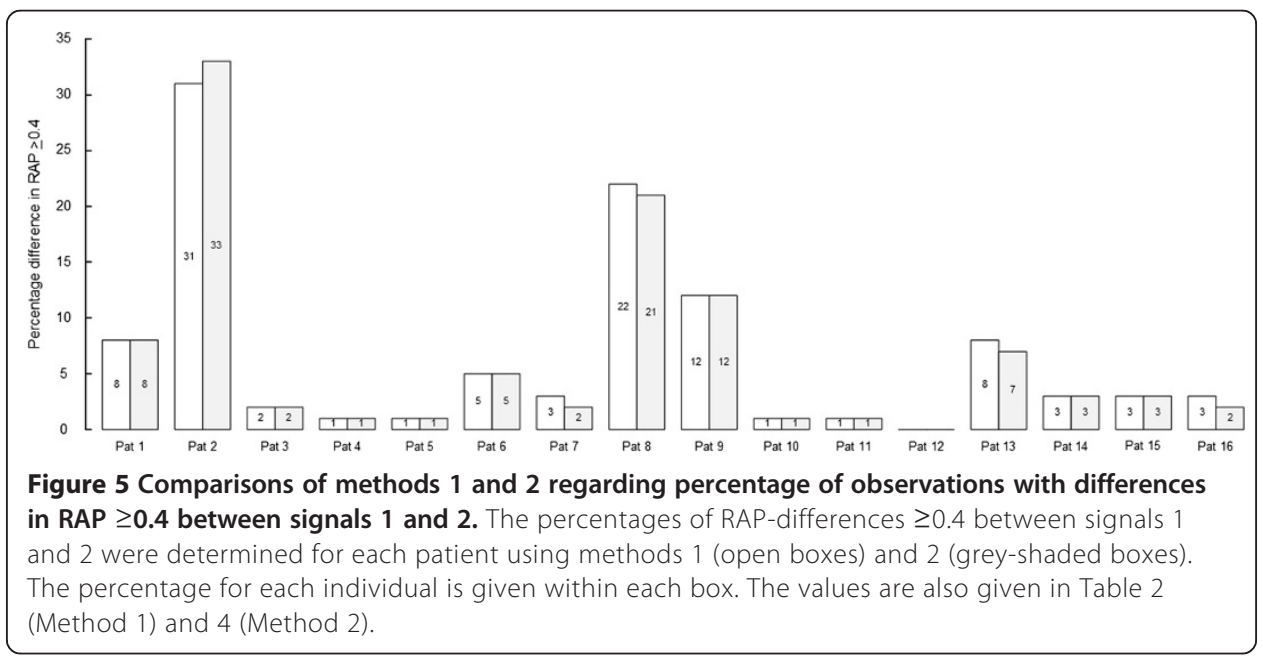


Table 5 Proportion of 6 -sec observations of RAP $<0.6$ in both signals (left), RAP $\geq 0.6$ in both signals (middle) and RAP $\geq 0.6$ in one signal while RAP $<0.6$ in another signal (right)

\begin{tabular}{|c|c|c|c|}
\hline Pat ID & $\begin{array}{l}\text { RAP }_{\text {SIGNAL } 1}<0.6 / \\
\text { RAP }_{\text {SIGNAL } 2}<0.6\end{array}$ & $\begin{array}{l}\operatorname{RAP}_{\text {SIGNAL } 1} \geq 0.6 / \\
\operatorname{RAP}_{\text {SIGNAL } 2} \geq 0.6\end{array}$ & $\begin{array}{c}\operatorname{RAP}_{\text {SIGNAL } 1_{1} \geq 0.6 / \text { RAP }_{\text {SIGNAL } 2}<0.6} \text { or } \\
\text { RAP }_{\text {SIGNAL } 1_{1}<0.6 / \text { RAP }_{\text {SIGNAL } 2} \geq 0.6}\end{array}$ \\
\hline 1 & 53,345 (73.1\%) & 14,269 (19.5\%) & $5,391(7.4 \%)$ \\
\hline 2 & 35,400 (84.0\%) & $498(1.2 \%)$ & $6,264(14.9 \%)$ \\
\hline 3 & $71,434(34.6 \%)$ & $114,056(55.2 \%)$ & $21,218(10.3 \%)$ \\
\hline 4 & $51,401(65.2 \%)$ & 22,404 (28.4\%) & 5,069 (6.4\%) \\
\hline 5 & $12,803(29.1 \%)$ & $29,420(67.0 \%)$ & 1,713 (3.9\%) \\
\hline 6 & 25,215 (57.5\%) & $13,893(31.7 \%)$ & $4,780(10.9 \%)$ \\
\hline 7 & 31,849 (25.1\%) & 85,127 (67.2\%) & 9,792 (7.7\%) \\
\hline 8 & 69,759 (46.6\%) & 47,354 (31.6\%) & $32,512(21.7 \%)$ \\
\hline 9 & $60,638(36.3 \%)$ & $80,763(48.3 \%)$ & $25,843(15.5 \%)$ \\
\hline 10 & $112,598(79.7 \%)$ & $23,769(16.8 \%)$ & 4,991 (3.5\%) \\
\hline 11 & 21,155 (35.5\%) & 35,341 (59.4\%) & $3,024(5.1 \%)$ \\
\hline 12 & 12,949 (10.5\%) & $108,783(88.2 \%)$ & $1,650(1.3 \%)$ \\
\hline 13 & 38,716 (71.2\%) & 8,457 (15.5\%) & 7,238 (13.3\%) \\
\hline 14 & $54,390(65.1 \%)$ & $23,122(27.7 \%)$ & $5,986(7.2 \%)$ \\
\hline 15 & $104,728(47.5 \%)$ & 94,725 (43.0\%) & $20,823(9.5 \%)$ \\
\hline 16 & 71,439 (63.6\%) & $33,122(29.5 \%)$ & 7,784 (6.9\%) \\
\hline
\end{tabular}

RAP: correlation coefficient (R) between the intracranial pressure (ICP) wave amplitude (A) and the mean ICP level (P).

amplitude is retrieved from the first harmonic of the power spectrum, hence providing an approximation of the amplitude [23]. In this present work, which compared RAP values, the mode of amplitude computation should not affect the results.

It can be discussed how many data points that should be used for computation of RAP. Most recent studies have determined RAP from 40 data points separated by 6 seconds over 4 min periods $[3,7-9,11]$. We used the same approach in this present study.

\section{Comparison of RAP scores from two simultaneous ICP signals}

Combining the present RAP findings with those of an earlier study [16], differences in RAP are smaller when comparing signals from similar ICP sensor types and larger when comparing signals from different ICP sensor types [16]. The largest differences were hence seen when monitoring from one solid - and one fluid ICP sensor.

The present findings extend our previous observations of marked differences in 4-min RAP when monitored simultaneously from two separate ICP sensors [16]. A difference in 4-min RAP $\geq 0.4$ was hence seen in $\geq 8 \%$ of observations in $5 / 16$ (31\%) patients. Determining the proportion of 4-min observations wherein the RAP was $\geq 0.6$ in one signal and $<0.6$ in another (above normal threshold level in one sensor and below in the other) revealed this setting in $>14 \%$ of scores in $4 / 16(25 \%)$ patients and $>9 \%$ of scores in another 4/16 (25\%) patients. The combination of above normal threshold level in one sensor and below in the other is of particular importance because it carries the potential to change the clinical decision making process.

Determining the RAP every 4 min period (presently referred to as method 1) carries the potential risk of introducing high variability in the recorded value. In an attempt to 


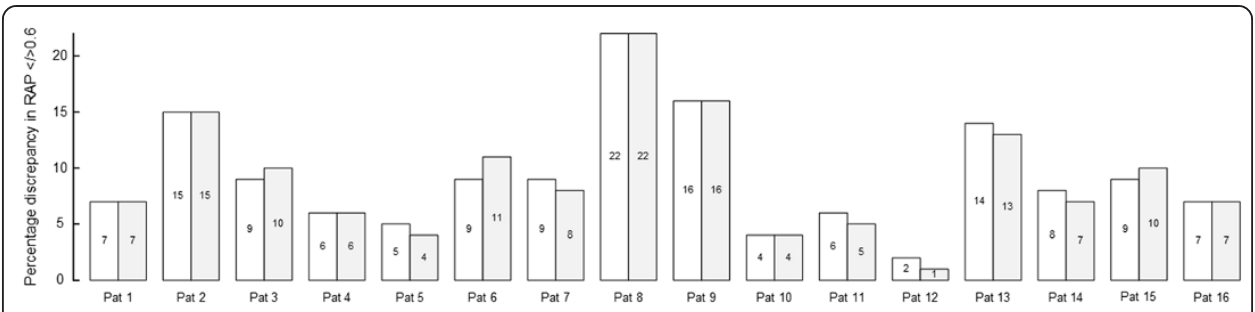

Figure 6 Comparisons of methods 1 and 2 regarding percentage of observations with discrepancy in RAP $</ \geq 0.6$ between signals $\mathbf{1}$ and 2 . The percentages of observations wherein one signal showed RAP $\geq 0.6$ while the other showed RAP $<0.6$ were determined for each patient using methods 1 (open boxes) and 2 (grey-shaded boxes). The percentage for each individual is given within each box. The values are also given in Table 3 (Method 1) and 5 (Method 2).

reduce the variation in calculated RAP, some clinicians chose to use a moving window with updates every 5-10 seconds [18]. Incorporating such a moving time window with updates every 5-10 seconds means that the 'oldest' averaged value drops out and the 'newest' drops in, thereby slightly altering the Pearson correlation coefficient every 5-10 seconds. Presently we have shown that incorporating such a moving time window with frequent updates of RAP (method 2) do not reduce the discrepancy in RAP between the two signals, as compared to updating the RAP every 4-min period (method 1).

Another approach to reduce the variability in RAP is to average scores over a long time period, e.g. 20-30 min. However, averaging scores works as filtering of the calculated values, and has the drawback of hiding information. Averaging over long time periods will thus extensively mask differences in RAP. This can be seen in Table 2, left, where the average of RAP scores over many hours of recording reveals only minor differences in the RAP. From a clinical perspective, such multi-hour average values are of limited interest. Since RAP is recommended for use in the surveillance of critically ill patients, short-term updates of RAP would be needed. Averaging over 20-30 minute periods has hence less relevance since marked clinical deterioration may happen over such long periods.

Obviously, it can be disputed which differences in RAP that have clinical significance. In this context, it should be remembered that ICP and ICP-derived indices such as RAP are used in the surveillance of critically ill patients. An erroneous measurement revealed to the physician/nurse may represent potential harm to the patient. In this present study cohort, one ICP sensor showed RAP $>0.6$ while the other showed RAP $<0.6$ in about $1 / 10$ observations (9-14\%) in 8/16 patients. Given the diagnostic importance of such an index in patient management, we consider such a difference of clinical significance.

In the present patients, ICP monitoring was done as part of patient surveillance. Therefore, monitoring was independent whether the EVD was open or closed, and independent the opening pressure of the EVD. Since the ICP sensors were placed close by within the brain, both ICP sensors would be similarly impacted independent of drainage through the EVD.

The RAP should be considered together with the mean ICP since RAP may become de-coupled from the mean ICP when mean ICP is very high $(>20-40 \mathrm{mmHg}$ ) [12]. It should be noted that mean ICP of the patients presented here was well below $20 \mathrm{mmHg}$, as recently reported for this same patient material [15]. 


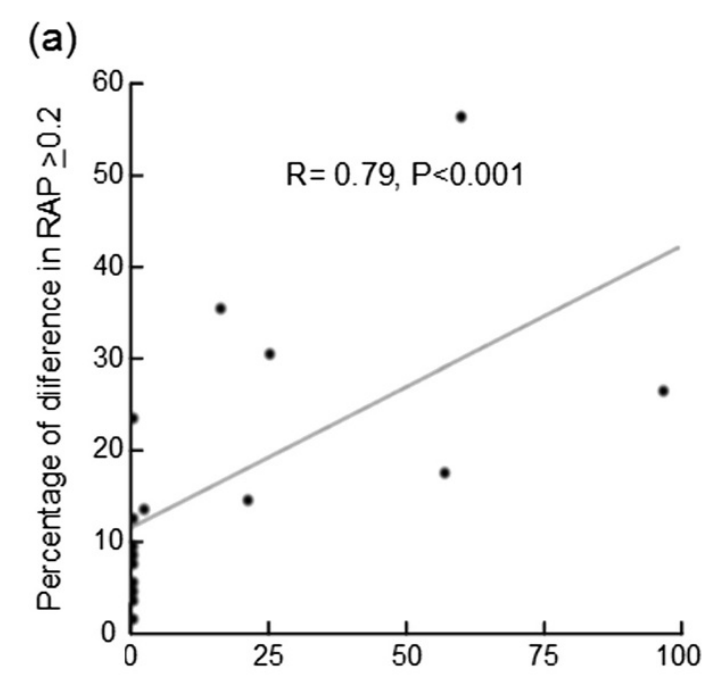

(b)

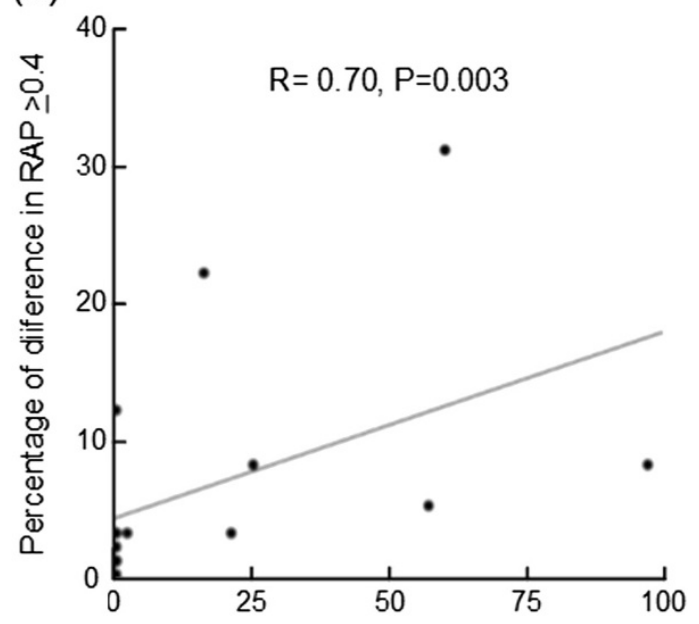

(c)

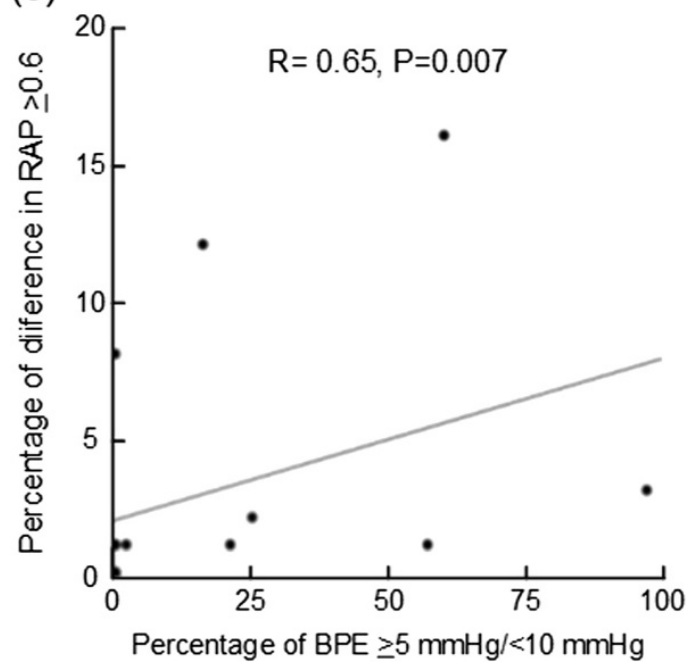

Figure 7 (See legend on next page.) 
(See figure on previous page.)

Figure 7 Correlation plots of percentages of baseline pressure errors (BPEs) and RAP [correlation coefficient (R) between the intracranial pressure (ICP) wave amplitude (A) and the mean ICP level (P)]-differences. The percentages of RAP-differences are plotted against the percentages of BPEs of various magnitudes for the 16 patients of the study. The percentages of BPEs of magnitudes $\geq 5 \mathrm{mmHg} /<10 \mathrm{mmHg}$ were plotted against differences in RAP of (a) $\geq 0.2$ or (b) $\geq 0.4$, and (c) $\geq 0.6$. The Spearman correlation coefficients are given suggesting significant correlation between percentages of BPEs and percentages of RAP-differences. The plots were created based on percentages provided in Table 4.

\section{Baseline pressure errors (BPEs)}

In 2006 Eide [13] described the phenomenon of baseline pressure errors (BPEs) when monitoring ICP simultaneously from two separate ICP sensors. The BPEs were manifested as marked differences in mean ICP combined with close to identity in ICP wave parameters such as the MWA [13]. In subsequent studies, BPEs could explain the differences in mean ICP observed when the two sensors were placed in different intracranial compartments $[24,25]$. By monitoring simultaneously from two separate ICP sensors, we have observed BPEs in solid sensors (Codman), air-pouch sensors (Spiegelberg) and fluid sensors (Edward's Life science) [14]; indeed, BPEs have been observed in every type of ICP sensors tested, the Raumedic Neurovent P [26,27], the Codman [28], the Camino [29] and the Spiegelberg [30] sensor. Our recent prospective observational study has confirmed that BPEs occur frequently in the clinical setting, and can be of a magnitude that may affect clinical management [15]. BPEs may also explain the abrupt shifts and drifts in the relationship between mean ICP and MWA that are observed when monitoring using merely one ICP sensor [31].

In an experimental study, Eide and Bakken [32], showed that solid ICP sensors are sensitive to electrostatic discharges (ESDs) and observed BPEs in the form of sudden pressure shifts. Pressure drifts were thus seen following ESDs. The BPEs were of a magnitude that could alter patient management in a clinical setting given similar changes in mean ICP. This observation has recently been confirmed by others [33]. The cause of BPEs may be different when recording from a fiber-optic ICP sensor, a solid sensor based on the whetstone bridge principle, or from an air-pouch type of ICP sensor. All technical components of an ICP monitoring system (sensor, cable, transducer, display) represent potential sites of origin of BPEs. When monitoring ICP through an EVD, BPEs may in addition be created by imperfect fluid connection caused by air bubbles and debris, or through movement of the sensor position (height) relative to the measurement site [14].

\section{Impact of BPEs on ICP-derived indices}

As illustrated in Figure 1, the BPEs were revealed as marked differences in mean ICP combined with close to identical ICP waveform. The largest differences in RAP were seen in patients $2,3,6,7,8,9,13$, and 15 (Table 3). In these very same patients, there was also a high frequency and severity of BPEs.

Given that BPEs cause alterations in mean ICP, it is to be expected that every pressure index wherein the mean ICP is incorporated also becomes affected. However, the differences in RAP are less pronounced than the differences in mean ICP. This is because the RAP also incorporates the ICP amplitude, which is resistant to BPEs. For 
this reason, indices solely based on static pressure measures such as the pressure reactivity index (PRx), which is the moving correlation between mean ICP and mean arterial blood pressure (mean ABP) [34], can be anticipated to be particularly susceptible to BPEs.

Due to the effect of BPEs on the RAP indices, its feasibility as a guide in the management of neurosurgical patients will be hampered. In our opinion, the divergence in RAP from the two sensors is of such an extent that we do not advocate the use of RAP in clinical practice. In contrast, since the ICP wave amplitude is unaffected by BPEs, the ICP wave amplitude is a robust parameter. Thus, in a recent study comparing the ICP wave amplitude, ICP wave slope, and RAP as measures of intracranial compliance in head injury patients, the ICP wave amplitude was found to be superior to the other parameters [21].

\section{Conclusions}

Simultaneous monitoring from two separate, close-by ICP sensors reveals significant differences in RAP that correspond to the occurrence of BPEs. As differences in RAP are of magnitudes that may alter patient management, we do not advocate the use of RAP as a guide in the management of neurosurgical patients.

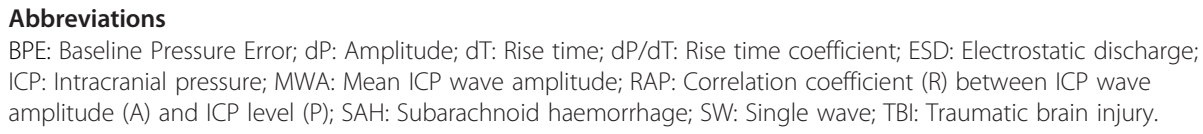

\section{Competing interests}

AS, TRM and WSO report no conflicts of interest. PKE has financial interest in the software company (dPCom A/S) that manufactures the software (Sensometrics ${ }^{\oplus}$ Software), which was used for digital recording of the continuous pressure signals in this study.

\section{Authors' contributions}

All authors contributed to conception and design, acquisition and interpretation of data. PKE contributed the bulk of the drafting of the manuscript and AS, TRM and WSO contributed with thorough editing of the manuscript. All authors have read and approved the final manuscript.

Received: 26 June 2014 Accepted: 17 July 2014

Published: 23 July 2014

\section{References}

1. Eide PK, Bentsen G, Sorteberg AG, Marthinsen PB, Stubhaug A, Sorteberg W: A randomized and blinded single-center trial comparing the effect of intracranial pressure and intracranial pressure wave amplitude-guided intensive care management on early clinical state and 12-month outcome in patients with aneurysmal subarachnoid hemorrhage. Neurosurgery 2011, 69(5):1105-1115.

2. Chesnut RM, Temkin N, Carney N, Dikmen S, Rondina C, Videtta W, Petroni G, Lujan S, Pridgeon J, Barber J, Machamer J, Chaddock K, Celix JM, Cherner M, Hendrix T: Global Neurotrauma Research Group. A trial of intracranial-pressure monitoring in traumatic brain injury. N Engl J Med 2012, 367(26):2471-2481. doi:10.1056/ NEJMoa1207363.

3. Czosnyka M, Pickard JD: Monitoring and interpretation of intracranial pressure. J Neurol Neurosurg Psychiatry 2004, 75(6):813-821.

4. Hutchinson PJ, Kolias AG, Czosnyka M, Kirkpatrick PJ, Pickard JD, Menon DK: Intracranial pressure monitoring in severe traumatic brain injury. BMJ 2013, 346:f1000.

5. Czosnyka M, Smielewski P, Timofeev I, Lavinio A, Guazzo E, Hutchinson P, Pickard JD: Intracranial pressure: more than a number. Neurosurg Focus 2007, 22(5):E10.

6. Czosnyka M, Price DJ, Williamson M: Monitoring of cerebrospinal dynamics using continuous analysis of intracranial pressure and cerebral perfusion pressure in head injury. Acta Neurochir (Wien) 1994, 126(2-4):113-119.

7. Kim DJ, Czosnyka Z, Keong N, Radolovich DK, Smielewski P, Sutcliffe MP, Pickard JD, Czosnyka M: Index of cerebrospinal compensatory reserve in hydrocephalus. Neurosurgery 2009, 64(3):494-501. discussion 501-492.

8. Schuhmann MU, Sood S, McAllister JP, Jaeger M, Ham SD, Czosnyka Z, Czosnyka M: Value of overnight monitoring of intracranial pressure in hydrocephalic children. Pediatr Neurosurg 2008, 44(4):269-279.

9. Timofeev I, Czosnyka M, Nortje J, Smielewski P, Kirkpatrick P, Gupta A, Hutchinson P: Effect of decompressive craniectomy on intracranial pressure and cerebrospinal compensation following traumatic brain injury. J Neurosurg 2008, 108(1):66-73.

10. Smith M: Monitoring intracranial pressure in traumatic brain injury. Anesth Analg 2008, 106(1):240-248. 
11. Weerakkody RA, Czosnyka M, Schuhmann MU, Schmidt E, Keong N, Santarius T, Pickard JD, Czosnyka Z: Clinical assessment of cerebrospinal fluid dynamics in hydrocephalus. Guide to interpretation based on observational study. Acta Neurol Scand 2011, 124(2):85-98.

12. Czosnyka M, Guazzo E, Whitehouse M, Smielewski P, Czosnyka Z, Kirkpatrick P, Piechnik S, Pickard JD: Significance of intracranial pressure waveform analysis after head injury. Acta Neurochir (Wien) 1996, 138(5):531-541. discussion 541-532

13. Eide PK: Comparison of simultaneous continuous intracranial pressure (ICP) signals from a Codman and a Camino ICP sensor. Med Eng Phys 2006, 28(6):542-549.

14. Eide PK, Holm S, Sorteberg W: Simultaneous monitoring of static and dynamic intracranial pressure parameters from two separate sensors in patients with cerebral bleeds: comparison of findings. Biomed Eng Online 2012, 11(1):66.

15. Eide PK, Sorteberg A, Meling TR, Sorteberg W: Baseline pressure errors (BPEs) extensively influence intracranial pressure scores: results of a prospective observational study. Biomed Eng Online 2014, 13(1):7.

16. Eide PK, Sorteberg W: An intracranial pressure-derived index monitored simultaneously from two separate sensors in patients with cerebral bleeds: comparison of findings. Biomed Eng Online 2013, 12:14

17. Eide PK: A new method for processing of continuous intracranial pressure signals. Med Eng Phys 2006, 28(6):579-587.

18. Budohoski KP, Czosnyka M, de Riva N, Smielewski P, Pickard JD, Menon DK, Kirkpatrick PJ, Lavinio A: The relationship between cerebral blood flow autoregulation and cerebrovascular pressure reactivity after traumatic brain injury. Neurosurgery 2012, 71(3):652-660. discussion 660-651.

19. Czosnyka M, Citerio G: Brain compliance: the old story with a new 'et cetera'. Intensive Care Med 2012, 38(6):925-927.

20. Eide PK, Sorteberg W: Diagnostic intracranial pressure monitoring and surgical management in idiopathic normal pressure hydrocephalus: a 6-year review of 214 patients. Neurosurgery 2010, 66(1):80-91.

21. Howells T, Lewen A, Skold MK, Ronne-Engstrom E, Enblad P: An evaluation of three measures of intracranial compliance in traumatic brain injury patients. Intensive Care Med 2012, 38(6):1061-1068.

22. Eide PK, Kerty E: Static and pulsatile intracranial pressure in idiopathic intracranial hypertension. Clin Neurol Neurosurg 2011, 113(2):123-128.

23. Holm S, Eide PK: The frequency domain versus time domain methods for processing of intracranial pressure (ICP) signals. Med Eng Phys 2008, 30(2):164-170.

24. Eide PK: Comparison of simultaneous continuous intracranial pressure (ICP) signals from ICP sensors placed within the brain parenchyma and the epidural space. Med Eng Phys 2008, 30(1):34-40.

25. Eide PK, Sorteberg W: Simultaneous measurements of intracranial pressure parameters in the epidural space and in brain parenchyma in patients with hydrocephalus. J Neurosurg 2010, 113(6):1317-1325.

26. Citerio G, Piper I, Chambers IR, Galli D, Enblad P, Kiening K, Ragauskas A, Sahuquillo J, Gregson B: Multicenter clinical assessment of the Raumedic Neurovent-P intracranial pressure sensor: a report by the BrainIT group. Neurosurgery 2008, 63(6):1152-1158. discussion 1158.

27. Stendel R, Heidenreich J, Schilling A, Akhavan-Sigari R, Kurth R, Picht T, Pietila T, Suess O, Kern C, Meisel J, Brock M: Clinical evaluation of a new intracranial pressure monitoring device. Acta Neurochir (Wien) 2003, 145(3):185-193. discussion 193.

28. Koskinen LO, Olivecrona M: Clinical experience with the intraparenchymal intracranial pressure monitoring Codman MicroSensor system. Neurosurgery 2005, 56(4):693-698. discussion 693-698.

29. Gelabert-Gonzalez M, Ginesta-Galan V, Sernamito-Garcia R, Allut AG, Bandin-Dieguez J, Rumbo RM: The Camino intracranial pressure device in clinical practice. Assessment in a 1000 cases. Acta Neurochir (Wien) 2006, 148(4):435-441.

30. Lang J-M, Beck J, Zimmermann M, Seifert V, Raabe A: Clinical evaluation of intraparenchymal spiegelberg pressure sensor. Neurosurgery 2003, 52(6):1455-1459.

31. Eide PK, Rapoport BI, Gormley WB, Madsen JR: A dynamic nonlinear relationship between the static and pulsatile components of intracranial pressure in patients with subarachnoid hemorrhage. J Neurosurg 2010, 112(3):616-625.

32. Eide PK, Bakken A: The baseline pressure of intracranial pressure (ICP) sensors can be altered by electrostatic discharges. Biomed Eng Online 2011, 10:75.

33. Andresen M, Juhler M, Thomsen OC: Electrostatic discharges and their effect on the validity of registered values in intracranial pressure monitors. J Neurosurg 2013, 119(5):1119-1124.

34. Eide PK, Sorteberg A, Bentsen G, Marthinsen PB, Stubhaug A, Sorteberg W: Pressure-derived versus pressure wave amplitude-derived indices of cerebrovascular pressure reactivity in relation to early clinical state and 12-month outcome following aneurysmal subarachnoid hemorrhage. J Neurosurg 2012, 116(5):961-971.

doi:10.1186/1475-925X-13-99

Cite this article as: Eide et al:: The effect of baseline pressure errors on an intracranial pressure-derived index: results of a prospective observational study. BioMedical Engineering OnLine 2014 13:99. 